MATEC Web of Conferences 6, 03005 (2013)

DOI: $10.1051 /$ matecconf/20130603005

(C) Owned by the authors, published by EDP Sciences, 2013

\title{
Combined NMR moisture, temperature and pressure measurements during heating
}

\author{
L. Pel ${ }^{1}$, S. Jaspers ${ }^{1}$, F. Pereira ${ }^{2,3}$, P. Pimienta ${ }^{2}$ and H. Carré ${ }^{3}$ \\ ${ }^{1}$ Department of Applied Physics, Eindhoven University of Technology, Eindhoven, \\ The Netherlands \\ 2 CSTB Centre Scientifique et Technique du Bâtiment, Paris, France \\ ${ }^{3}$ SIAME, University of Pau and Pays de l'Adour, Anglet, France
}

\begin{abstract}
For model validation, quantitative measurements of the evolution of moisture, temperature, and pressure distributions in time are needed. For this purpose, we have developed an NMR setup to measure the moisture transport in heated building materials. The measured combined moisture content and temperature profiles give a unique insight in the moisture transport and dehydration kinetics inside concrete during fire. These measurements give the first quantitative proof for the build-up of a moisture peak due to the vapor pressure build-up. In this study we have also combined for the first time the measurement of the moisture and temperature profiles with the measurement of the pressure at one position, which show that the pressure build up is directly related to the moisture profiles.
\end{abstract}

\section{INTRODUCTION}

The moisture content and transport in concrete, together with the vapor permeability, is presumed one of the most influential factors for fire spalling. It is generally assumed that below a certain moisture content of approximately 5 vol.\% no spalling will occur [1,2]. Numerous heat and mass transfer models have been used to predict the moisture transport and its consequences on the strength and permeability of the concrete [3-8]. However, these models are only of use if they can be validated. For model validation, quantitative measurements of the evolution of moisture, temperature, and possibly pressure distributions in time are needed.

A few (indirect) observations of the moisture content in concrete have been reported in literature. Water has been observed bleeding from the cool side of a concrete wall, indicating that the surface layer is saturated [9]. Jansson et al. report a method in which a concrete cube is heated from one side. By splitting the heated cube in half, a layer with a relative higher saturation is observed by a discoloration of the concrete [10]. In this way, the sample is destroyed by the act of measurement itself. It will be extremely laborious to obtain a time evolution of the moisture content. Each time increment would also be from a different concrete sample. Experimentally determined moisture contents with a spatial resolution of about $20 \mathrm{~cm}$ were presented in paper by Ichikawa et al. [11]. However, the obtained resolution is far too low to validate the model results.

With a dedicated NMR fire spalling setup it is possible to measure both the moisture and temperature distributions non-destructively. This setup has been extensively tested in previous experiments on benchmark materials such as fired-clay brick, calcium silicate brick, and gypsum [12-14]. For the measurements on concrete it is necessary to have a validated measurement technique since the moisture content of concrete in equilibrium with, e.g., $50 \% \mathrm{RH}$, is typically in the range of $10^{-3} \mathrm{~m}^{3} \mathrm{~m}^{-3}$. Although the low moisture content is challenging the capabilities of the NMR setup, it was possible to measure the

This is an Open Access article distributed under the terms of the Creative Commons Attribution License 2.0, which permits unrestricted use, distribution, and reproduction in any medium, provided the original work is properly cited. 


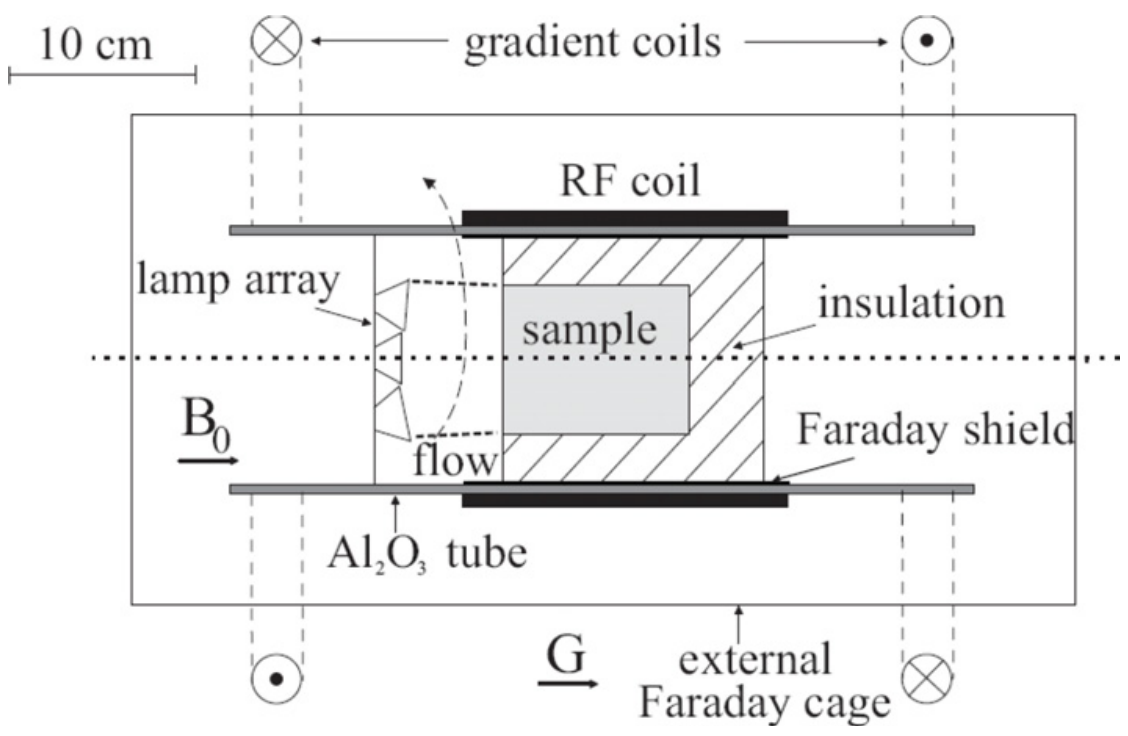

Figure 1. Schematic diagram of the NMR setup. The setup has a cylindrical symmetry. A whole-body 1.5 T MRI scanner provides the main magnetic field. An anti-Helmholtz coil configuration provides a constant magnetic field gradient G. A bird-cage RF coil with a diameter of $140 \mathrm{~mm}$ is used for both sending the RF pulses and receiving the NMR signal. An array of four $100 \mathrm{~W}$ halogen lamps is used to heat the sample. The sample has a diameter of $80 \mathrm{~mm}$ and a length of $100 \mathrm{~mm}$. It is thermally insulated and positioned inside the bird-cage coil.

moisture transport in concrete. In this study we have also for the first time combined the measurement of the moisture and temperature profile with the measurement of the pressure in the sample. We will first discuss the NMR, followed by a discussion of the measurements.

\section{NMR SETUP}

\subsection{NMR basics}

Almost all nuclei have a magnetic dipole moment, resulting from their spin-angular momentum. One can think of a nucleus as a charged sphere spinning around its axis, which corresponds to a current loop, generating a magnetic moment. In a Nuclear Magnetic Resonance (NMR) experiment the magnetic moments of the nuclei are manipulated by suitably chosen electromagnetic radio frequency (RF) fields. The frequency of the resonance conditions is given by:

$$
f_{l}=\frac{\gamma}{2 \pi} B_{o}
$$

where $\mathrm{f}_{l}$ is so-called Larmor frequency $[\mathrm{Hz}], \mathrm{B}_{\mathrm{o}}[\mathrm{T}]$ the externally applied static magnetic field and $\gamma$ is the gyromagnetic ratio which is dependent on the type of nucleus (for ${ }^{1} \mathrm{H} \gamma / 2 \pi=42.58 \mathrm{MHz} \mathrm{T}^{-1}$ ). Because of this condition the method can be made sensitive to only hydrogen and therefore to water, in contrast to the attenuation methods. When a known magnetic field gradient is applied, the constant magnetic field $\mathrm{B}_{\mathrm{o}}$ in the resonance condition (Eq. (1)) has to be replaced by the spatially varying magnetic field B:

$$
B(x)=B_{o}+G x
$$

where $\mathrm{G}\left[\mathrm{T} \mathrm{m}^{-1}\right.$ ] (see Fig. 1) is the magnetic field gradient and $\mathrm{x}$ is the position of the precessing magnetic moment. The resonance condition is then spatially dependent. Therefore the moisture content at different positions $\mathrm{x}$ in the sample can be measured by varying the resonance frequency $\mathrm{f}$ without 
moving the sample. In a pulsed NMR experiment the orientation of the moments of the spins are manipulated by short RF pulses at the resonance frequency. The amplitude of the resulting signal emitted by the nuclear spins, the so-called 'Hahn spin-echo' signal is proportional to the number of nuclei taking part in the experiment. The spin-echo signal also gives information about the rate at which this magnetic excitation of the spins decays. The system will return to its magnetic equilibrium by two mechanisms: interactions between the nuclei themselves, causing the so-called spin-spin relaxation, and interactions between the nuclei and their environment, causing the so-called spin-lattice relaxation. Assuming that both mechanisms give rise to a single exponential relaxation and that spin lattice relaxation is much slower then the spin-spin relaxation, the magnitude of the NMR spin-echo signal is given by:

$$
S=\rho \exp \left(-\frac{t_{E}}{T_{2}}\right)\left[1-\exp \left(-\frac{t_{R}}{T_{1}}\right)\right] .
$$

In this equation $\rho$ is the proton density, $\mathrm{T}_{1}$ the spin-lattice relaxation time, $\mathrm{t}_{\mathrm{R}}$ the repetition time of the spin-echo experiment, $T_{2}$ the spin-spin or transverse relaxation time and $t_{\mathrm{E}}$ the so-called spin-echo time. Obviously small $\mathrm{T}_{2}$ values lead to a decrease of the spin-echo signal, whereas, on the other hand, small $\mathrm{T}_{1}$ values are preferred, as this parameter limits the repetition time (usually $\mathrm{t}_{\mathrm{R}} \approx 4 \mathrm{~T}_{1}$ ) and hence the rate at which the moisture profiles can be scanned. Water in a porous building material has typically a relaxation time $\mathrm{T}_{1}$ in the order of $0.1-0.5 \mathrm{~s}$.

In porous materials $T_{2}$ will be decreased strongly with respect to that of 'pure' water, due to surface relaxation at the pore walls. In many common porous building materials, like fired-clay brick or concrete, usually large amounts of paramagnetic ions (e.g., Fe) are present. This complicates the NMR measurements by two effects. First, due to the large susceptibility of the porous material, the transverse relaxation time $T_{2}$ will be drastically decreased. Secondly, the variations in local magnetic susceptibility will broaden the resonance line and thereby limit the spatial resolution. The relaxation can give information about the distribution of pore sizes in a porous material [12].

For the experiments described in this study a specially designed NMR scanner was used. This instrument was especially designed for quantitative measurements of moisture in porous materials with short transverse relaxation $\left(T_{2}\right)$ times (unlike standard Magnetic Resonance Imaging (MRI), which is generally used in a qualitative way). The machine makes use of the magnet of a whole body MRI scanner (Gyroscan, Philips) which operates at a main field of 1.5 Tesla corresponding to a frequency of $63.9 \mathrm{MHz}$. The setup is placed within the scanner and a schematic diagram is given in Fig. 1 . Two Helmholtz coils provide the magnetic field gradient $G$ in the direction of $B_{0}$. The gradient has a magnitude of $86.5 \mathrm{mT} \mathrm{m}^{-1}$, providing a spatial resolution of the order of $4 \mathrm{~mm}$.

A home built birdcage coil with a diameter of $140 \mathrm{~mm}$ is used for applying the RF pulses, and receiving the NMR signal from the sample. A birdcage coil is used because it generates a homogeneous $\mathrm{B}_{1}$-field perpendicular to the sample in order to generate spin-echos. Therefore, the coil can be placed parallel to the main magnetic field providing optimal use of the available space inside the bore. The sample is heated with 4 halogen lamps. The reflectors of these lamps are gold plated to ensure maximum reflection of the infra-red radiation. The sample is placed inside the birdcage coil and is thermally insulated using mineral wool in order to create a 1D experiment.

\subsection{Signal calibration}

In order to obtain quantitative moisture content, the NMR signal must be calibrated against the moisture content of the concrete. For this purpose, the NMR signal was measured with the same settings as during the experiments. A sample was initially vacuum saturated, after which it was dried slowly to keep the moisture homogeneously distributed throughout the sample. Mass and signal were both recorded throughout the drying process. The NMR signal is shown as a function of the normalized sample mass in Fig. 2. For mass ratios between 1 and 0.94 a linear relation between the NMR signal and the moisture content is obtained. This indicates that the NMR signal can be directly related to the amount of free 


\section{MATEC Web of Conferences}

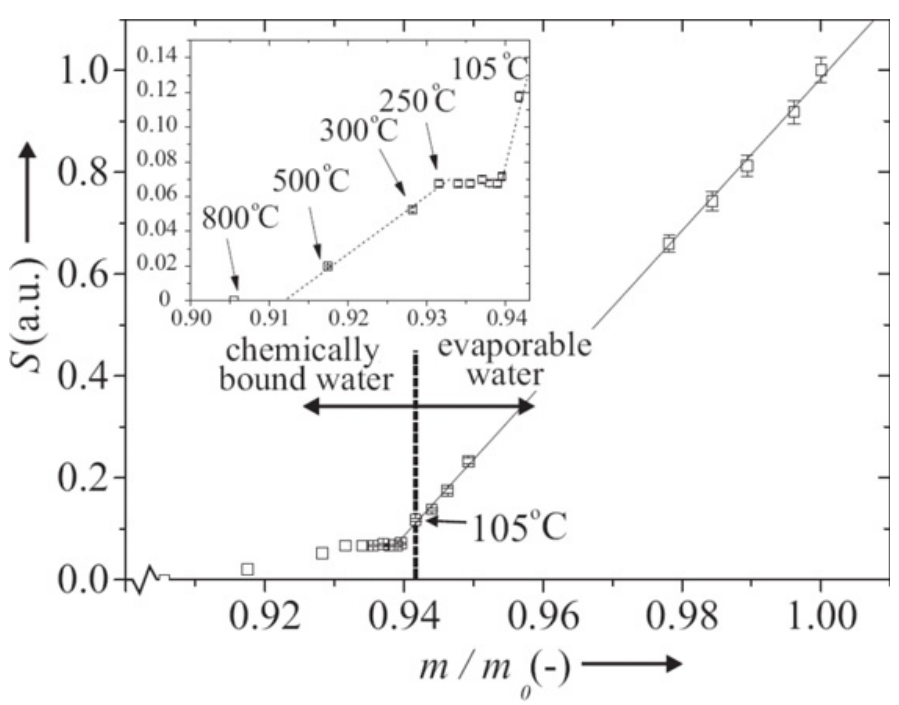

Figure 2. NMR signal as a function of normalized sample mass during a static isothermal calibration. The signal varies linearly with the amount of free moisture (solid line, $\mathrm{m} / \mathrm{m}_{0}>0.94$ ). The calibration of the non-evaporable moisture content (hydrated water) is shown in the inset. The temperatures indicate the temperature up to which the sample was heated.

moisture present in the concrete. After the sample was dried at ambient temperatures a further decrease in moisture content (chemically bound) was obtained by heating the sample up to a certain constant temperature above $100^{\circ} \mathrm{C}$.

After the sample had reached a constant mass, the sample was slowly cooled down to room temperature under a $0 \% \mathrm{RH}$ atmosphere. Thereby preventing any increase in moisture content or rehydration of the concrete. Then, at room temperature the NMR signal and mass were both measured. The results are shown in the inset of Fig. 2. A decrease in both signal and mass is observed for temperatures from $105^{\circ} \mathrm{C}$ to $130^{\circ} \mathrm{C}$. For the drying temperatures of $130{ }^{\circ} \mathrm{C}$ to $250{ }^{\circ} \mathrm{C}$ the mass of the sample decreases indicating that chemically bound moisture is lost from the concrete. However, the NMR signal remains constant. For temperatures above $300^{\circ} \mathrm{C}$ again both the signal as well as the moisture content decrease, until all signal is lost at a temperature between 500 and $800^{\circ} \mathrm{C}$. From this drying experiment we can conclude that the NMR signal is linearly related to the free moisture content. Furthermore, the NMR setup is capable of measuring a signal from the chemically bound moisture content. Unlike in, e.g., gypsum there is no linear relation between the chemically bound moisture content and the NMR signal. In fact, between 120 and $170^{\circ} \mathrm{C}$ no decrease in NMR signal is observed, although the mass of the sample decreases. At a temperature of $800{ }^{\circ} \mathrm{C}$ the concrete sample is completely dehydrated, resulting in zero NMR signal. In the future we will try to include the chemically bound moisture, but for now we will solely concentrate on the free moisture content.

\section{EXPERIMENTAL RESULTS}

\subsection{Sample preparation}

The cylindrical concrete sample used in this study were drilled from larger cast blocks of $400 \times 100 \times$ $100 \mathrm{~mm}$ and have a diameter of $80 \mathrm{~mm}$, and a length of $100 \mathrm{~mm}$. The concrete has a strength class of C40, with a water cement ratio of 0.5 . The details of the mix are given in Table 1.The concrete blocks were stored under water for three months before the samples were drilled. After drilling, the sample was 
Table 1. Concrete (C40) mix design.

\begin{tabular}{lc}
\hline Constituent & Amount $\left[\mathrm{kgm}^{-3}\right]$ \\
\hline CEM I 32.5 R & 350 \\
\hline water & 175 \\
\hline W/C ratio & 0.5 \\
\hline sand $(0.125-0.250)$ & 127 \\
\hline sand $(0.250-0.500)$ & 217 \\
\hline sand $(0.500-1)$ & 217 \\
\hline sand $(1-2)$ & 253 \\
\hline sand $(2-4)$ & 380 \\
\hline gravel $(4-8 \mathrm{~mm})$ & 614 \\
\hline Total & 2330 \\
\hline
\end{tabular}
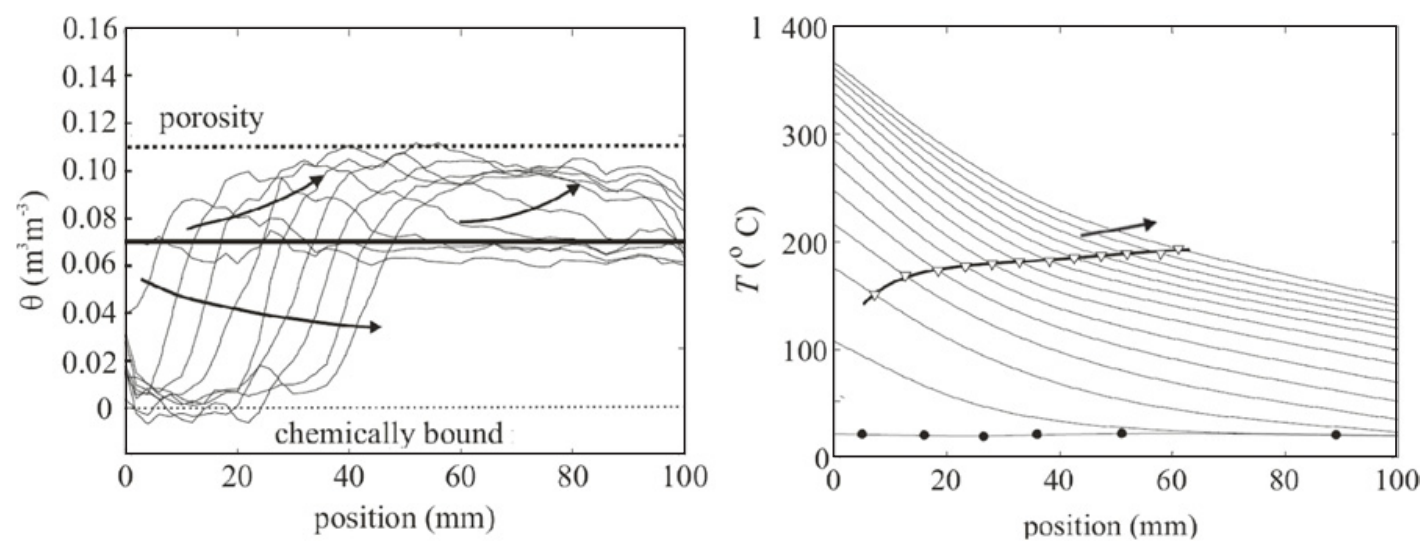

Figure 3. Moisture (left) and temperature profiles (right) of a concrete sample heated from one side at $12 \mathrm{kWm}^{-2}$. At the start of the experiment the sample was equilibrated at $97 \% \mathrm{RH}$, which corresponds to a moisture content of approximately $0.07 \mathrm{~m}^{3} \mathrm{~m}^{-3}$. The profiles are shown for every 8.5 minutes. The temperature at the boiling front is indicated.

first dried, after which it was equilibrated at 97\% RH. The sample was one year old at the time of the experiment. Before the experiment, the samples were pressed in a PTFE holder to seal all sides except for the heated surface. In this way the moisture transport is limited to one dimension. The flow of heat is limited to one dimension by insulating the sample using mineral wool $(4 \mathrm{~cm})$. Numerical simulations indicate that the radial heat flow is limited to $10 \%$ of the longitudinal heat flow.

\subsection{Measured profiles}

At the start of the experiment the concrete sample was first equilibrated at $97 \% \mathrm{RH}$, corresponding to a free moisture content of $0.07 \mathrm{~m}^{3} \mathrm{~m}^{-3}$. The moisture and temperature profiles are shown in Fig. 3. The moisture profiles are normalized with respect to the first moisture profiles (bold line). As the surface $(0 \mathrm{~mm})$ is heated the temperature increases after 8.5 minutes to above $100{ }^{\circ} \mathrm{C}$. The free moisture at the surface will boil and we can observe a boiling front developing at the surface. As the heating continues, the boiling front moves further into the material. To the left of the boiling front no free moisture content is measured. To provide a complete overview, we have chosen to include the signal originating from the chemically bound moisture. Therefore, the zero free moisture content level is indicated by $\theta=0$. However, it is possible for the signal to decrease below $\theta=0$, corresponding to loss of chemically 


\section{MATEC Web of Conferences}
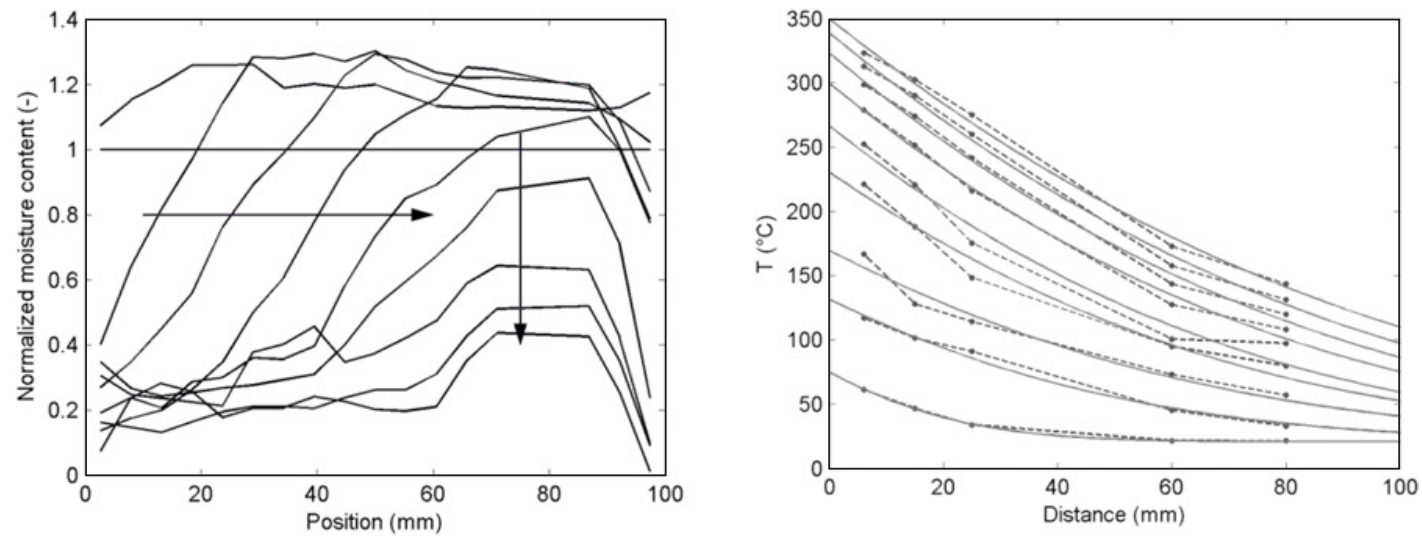

Figure 4. Moisture (left) and temperature profiles (right) of a concrete sample heated from one side at $12 \mathrm{kWm}^{-2}$. At the start of the experiment the sample was capillary saturated. The profiles are shown for every 14 minutes.

bound moisture. The measured temperature profiles indicate that the boiling and dehydration processes have no significant influence on the heat transport. In previous heating experiments on fired-clay brick and calcium silicate brick a clear inflection in the temperature profiles was observed either caused by boiling and/or dehydration [14]. In this experiment, the moisture content of the concrete is relatively low and hence both the heat capacity, and thermal conductivity of the sample are dominated by the concrete heat capacity and conductivity. As the boiling front moves further into the sample we can observe a significant increase in moisture content to the right of the boiling front. However, as the boiling front progresses the moisture content does not increase above, approximately $0.11 \mathrm{~m}^{3} \mathrm{~m}^{-3}$. This moisture content corresponds to the porosity of the concrete sample (indicated by the dashed line). The moisture content in this region cannot increase any further, because the material is saturated. To our knowledge this is the first direct and quantitative proof of so-called moisture clogging in concrete.

An explanation for the increase in moisture content can be found in the local vapor pressure at the boiling front. In Fig. $3 \mathrm{~b}$ the temperature at the boiling front is indicated. The temperature at the front increases from approximately $160^{\circ} \mathrm{C}$ to $195^{\circ} \mathrm{C}$. The saturated vapor pressures corresponding to these temperatures are 0.7 and $1.4 \mathrm{MPa}$ respectively, compared to $0.1 \mathrm{MPa}$ atmospheric pressure. The increase above $100{ }^{\circ} \mathrm{C}$ (atmospheric boiling point) indicates that there is a local increased vapor pressure. The vapor pressure is assumed to be equal to the saturated vapor pressure. As a result of these high vapor pressures the vapor released at the boiling front is advected both towards the heated surface as well as the back of the sample. A temperature gradient is present across the sample. As a result the saturated vapor pressure immediately to the right of the boiling front is lower. The vapor advected to the back of the sample, will therefore condensate, resulting in an increase of the free moisture content in the region to the right of the boiling front, i.e., the so-called moisture clogging. The vapor advected towards the surface is able to exit the sample.

\subsection{Pressure measurement}

To provide additional information we have also performed measurements in which we for the first time combined the NMR measurements of the moisture profile, temperature profiles with pressure measurements using a gauge embedded in the concrete. Here we have used an embedded CSTB pressure gauge as first proposed by Kalifa et al. [15]. This pressure gauge consists of a sintered metal plate in a cup which and was positioned at the main axis of the cylinder at a distance of $40 \mathrm{~mm}$ from the heated surface. In these preliminary experiments we have started with a capillary saturated sample. Although it was found that the pressure gauge disturbed the NMR measurements, after correction the moisture 

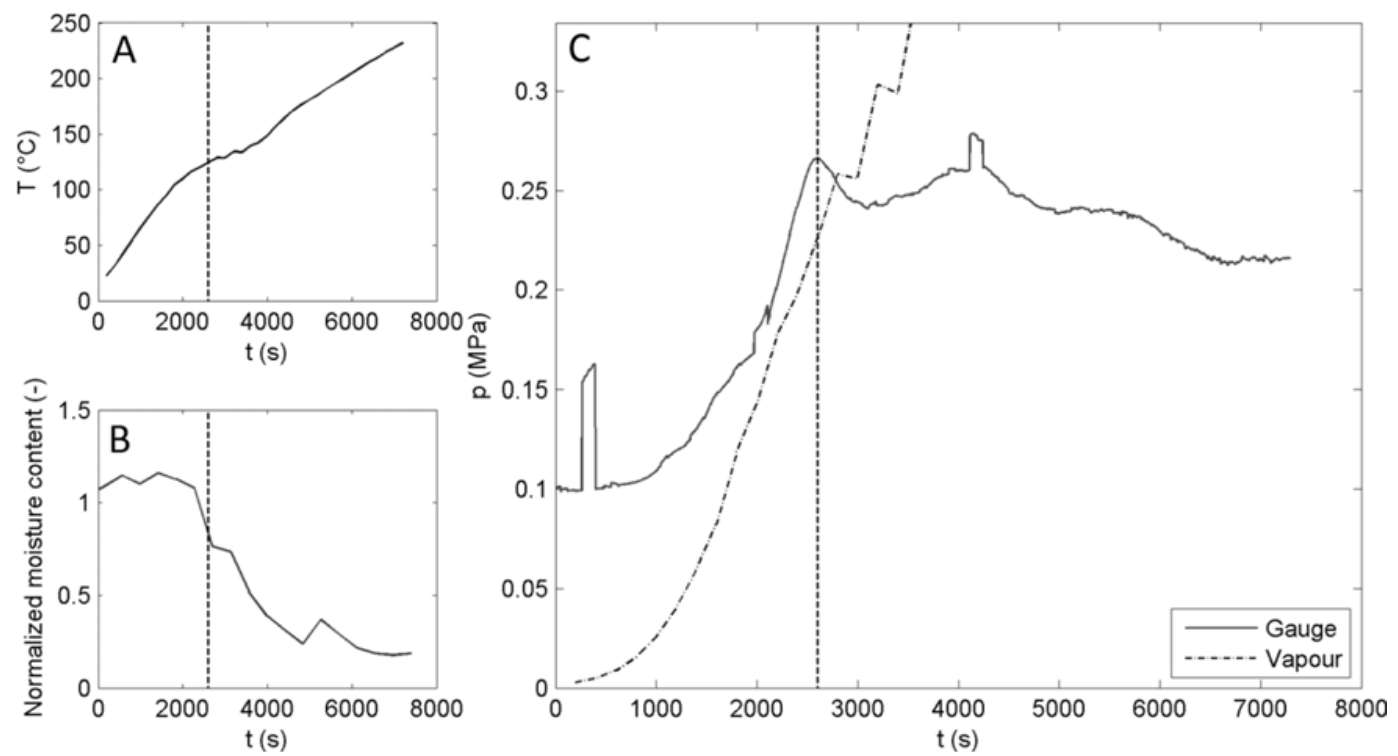

Figure 5. The results of combined measurements of the moisture profile, temperature and pressure as measured by an embedded pressure Gauge. Indicated are the temperatures (A) at the position of the pressure gauge $(40 \mathrm{~mm})$ as measured by the thermocouple in the pressure gauge, the moisture content at the position of the gauge (B) and the pressure as measured by the Gauge (C). The dashed line is the saturated vapor pressure as calculated from the temperature. Note that the pressure scale is chosen so that $0.1 \mathrm{MPa}$ corresponds to the saturated vapor pressure at $100{ }^{\circ} \mathrm{C}$ (or: $0.1 \mathrm{MPa}$ is atmospheric pressure).

profiles clearly show a receding boiling front entering the material as can be seen in Figure 4. The corresponding pressures as measured by the pressure gauge located at $40 \mathrm{~mm}$ from the top are given in Figure 5 .

As can be seen from Figure 4A a small plateau, i.e., around $2500 \mathrm{sec}$, is observed in the temperature as the boiling front passes the pressure gauge (fig 4B). This can be contributed to the energy consumption due to vaporization of the water $[15,16]$. As can be seen indeed the highest pressure is measured as the boiling front passes, indicating a local thermal equilibrium. Moreover the pressure as measured follows the saturated vapor pressure curve.

\section{CONCLUSION}

This study shows that NMR is a very powerful tool in providing accurate moisture and temperature profiles. It is possible to measure non-destructively the moisture distributions with NMR inside building materials during heating. The moisture profiles can be measured with a resolution of 4-5 $\mathrm{mm}$, while the sample is heated with a radiative heat flux of approximately $12 \mathrm{~kW} \mathrm{~m}^{-2}$. The measurements give the first quantitative proof for the buildup of a moisture peak. While all moisture transport models predict the existence of such a peak, these predictions were never validated with moisture profile measurements. Moreover, with this setup for the first time the combined measurement of moisture, temperature and vapor pressure could be performed. These preliminary measurements indicate that the vapor pressure measured follows the saturated vapor pressure curve.

Part of this research was supported by the Dutch Technology Foundation (STW), and TNO Built Environment and Geosciences. 


\section{MATEC Web of Conferences}

\section{References}

[1] C.E. Majorana, et al., An approach for modelling concrete spalling in finite strains. Mathematics and Computers in Simulation 80 (8), 1694-1712, 2010.

[2] P.C. Aitcin, Cements of yesterday and today - concrete of tomorrow. Cement and Concrete Research 30 (9), 1349-1359, 2000

[3] G.N. Ahmed and J.P. Hurst. Modeling pore pressure, moisture, and temperature in high-strength concrete columns exposed to fire. Fire Technology 35 (3), 232-262, 1999.

[4] D. Gawin, et al., Modelling of hygro-thermal behaviour of concrete at high temperature with thermo-chemical and mechanical material degradation. Computer Methods in Applied Mechanics and Engineering 192 (13-14), 1731-1771, 2003.

[5] B.A. Schrefler, et al., Thermo-hydro-mechanical modelling of high performance concrete at high temperatures, Engineering Computations 19 (7), 787-819, 2002.

[6] Benard, R.E et al, Boiling in porous media: Model and simulations, Transport in Porous Media 60(1) 1-31, 2005.

[7] C.L.D. Huang. Multiphase moisture transfer in porous-media subjected to temperature-gradient. International Journal of Heat and Mass Transfer 22 (9), 1295-1307, 1979.

[8] B.A. Schrefler, Multiphase flow in deforming porous material. International Journal for Numerical Methods in Engineering 60 (1), 27-50, 2004.

[9] J.C. Mindeguia, et al., Temperature, pore pressure and mass variation of concrete subjected to high temperature - experimental and numerical discussion on spalling risk., Cement and Concrete Research 40, 477-487, 2009.

[10] R Jansson et al., Fire spalling - the moisture effect, 1st International workshop on concrete fire spalling due to fire exposure, 2009.

[11] Y. Ichikawa et al., Prediction of moisture migration and pore pressure build-up in concrete at high temperatures. Nuclear Engineering and Design 228 (1-3), 245-259, 2008.

[12] G.H.A. vander Heijden, NMR imaging of moisture inside heated porous building materials, $\mathrm{PhD}$ thesis, Eindhoven University of Technology, the Netherlands (2011).

[13] G.H.A. vander Heijden et al., One-dimensional scanning of moisture in heated porous building materials with nmr. Journal of Magnetic Resonance 208 (2), 235-242, 2011.

[14] G.H.A. vander Heijden et al., Non-isothermal drying of fired-clay brick, an nmr study., Chemical Engineering Science 64 (12), 3010-3018, 2009.

[15] P. Kalifa, F.D. Menneteau, D. Quenard. Spalling and pore pressure in HPC at high temperatures, Cement and Concrete Research 30(12), 1915-1927, 2000.

[16] Mindeguia J.C., Pimienta P., I. Hager and H. Carre, Influence of the water content on the gas pressure in concretes at high temperatures, In Concrete spalling due to fire exposure, RILEM publication S.A.R.L., 2011. 